\title{
A SYSTEM OF MULTIGAP PROPORTIONAL WIRE CHAMBERS
}

W. BELL, L. DUMPS, H. G. FISCHER, H. FREHSE, F. GAGLIARDI, B. HECK, P. G. INNOCENTI, G. MAURIN, L. NAUMANN, A. NORTON, F. PIUZ,

M. SCIRÈ and O. ULLALAND

CERN, Geneva, Switzerland

\begin{abstract}
A set of proportional chambers has been constructed for a multiparticle spectrometer at the CERN Intersecting Storage Rings (ISR). The detector consists of 73000 wires and covers an effective area of $300 \mathrm{~m}^{2}$. In this paper we will describe the design and performance of this detector.
\end{abstract}

\section{Introduction}

The split field magnet detector ${ }^{1-3}$ ) (SFMD) is a multiparticle spectrometer adapted to the kinematics of colliding beams. It became available for physics at the ISR in 1973 (fig. 1). The spectrometer was designed to accept a typical event where the forward direction is densely populated by high momentum secondaries, whereas the particles in the central region mainly have momenta below $500 \mathrm{MeV} / c$.

After the first exploratory experiments, with the study of diffraction and single particle exchange reactions, the physics emphasis was shifted to large transverse momentum reactions and lepton production. These reactions have a special topology, and can be characterized by a high multiplicity and a strong clustering of the secondary particles in the central region as well as in the forward direction.

From these experiments it became evident that the detector, together with the accompanying software, which had been designed for the diffraction-like events, were less suited for the physics of current interest (fig. 2). This was mainly due to insufficient redundancy for pattern recognition and reconstruction of curved trajectories in the presence of several particles, secondary vertices and background.

It became therefore clear that the spectrometer had to match the changes in physics aims, and it was decided to up-grade the SFM detector.

\section{The upgraded SFM detector}

The basic features of the SFM detector have been kept, since the system of proportional chambers has proven to be an excellent device for event selection and background separation. It is self-triggering and can stand an event rate of about $1 \mathrm{MHz}$. The reliability and stability have also been very good.

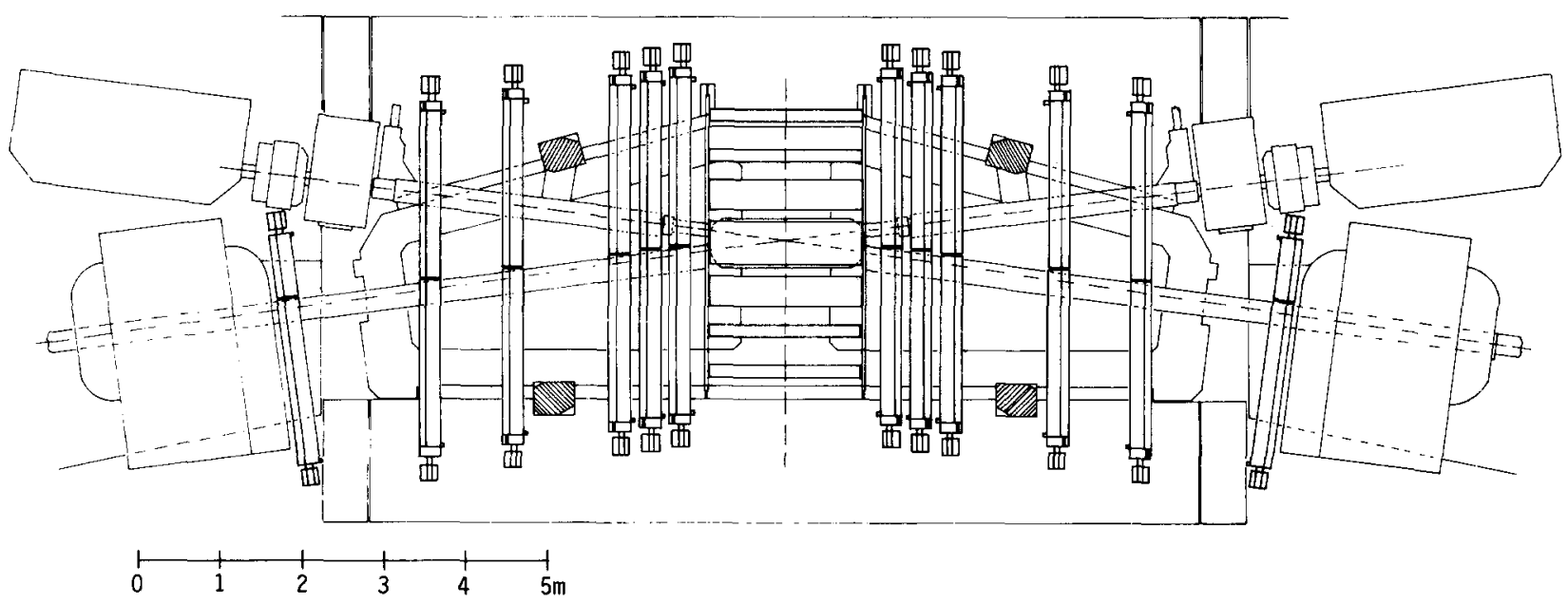

Fig. 1. Schematic view of the old detector layout. 


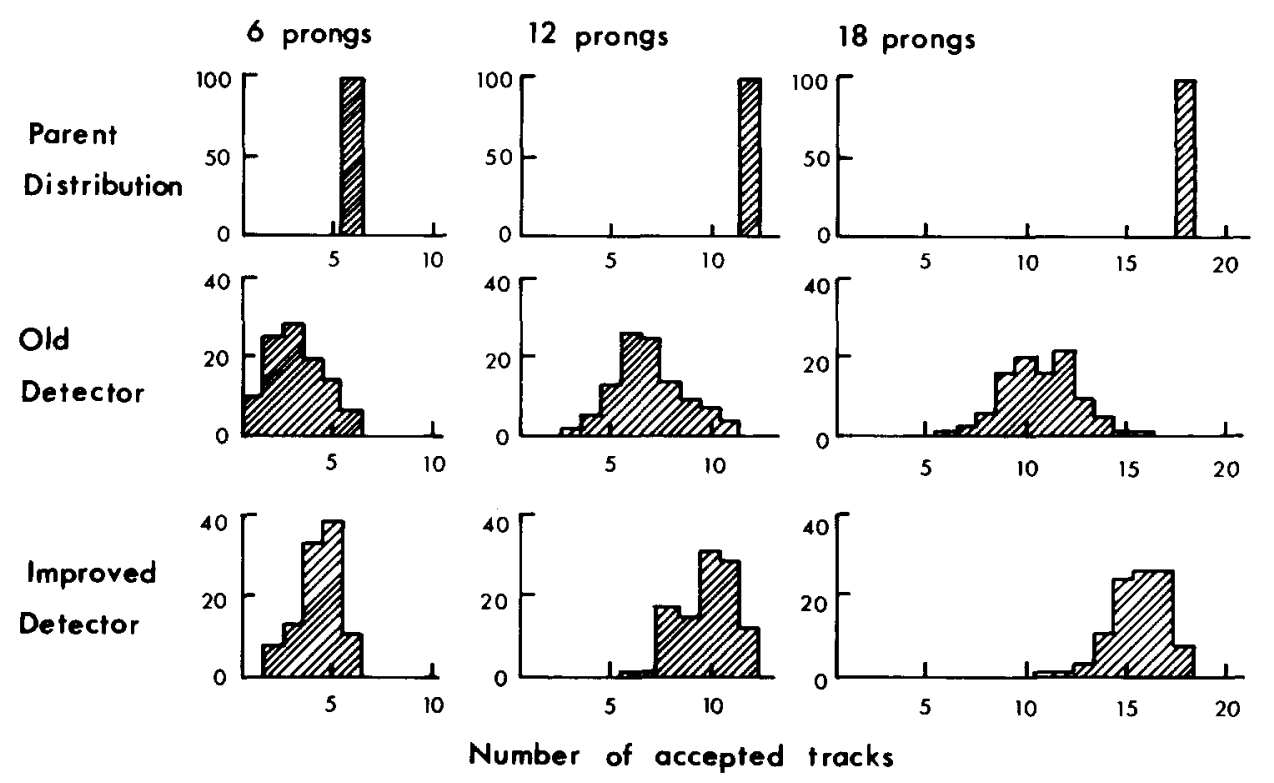

Fig. 2. The number of accepted tracks in the old and the new detector as compared to an $N$-prong event, which is distributed flat in rapidity and isotropically production angle. The rapidity $y=\frac{1}{2} \ln \left(E+p_{\|} / E-p_{\mid !}\right)$and the c.m. production angle $\phi$ is the angle between $p_{\perp}$ and the beam plane.

The improved detector (fig. 3) can be divided into two parts:

a) A central vertex detector around the interaction region. This part consists of essentially eight densly packed chamber units with up to eleven wireplanes per unit. The central part, $12 \mathrm{~m}^{3}$, is covered by an effective detection area of $145 \mathrm{~m}^{2}$.
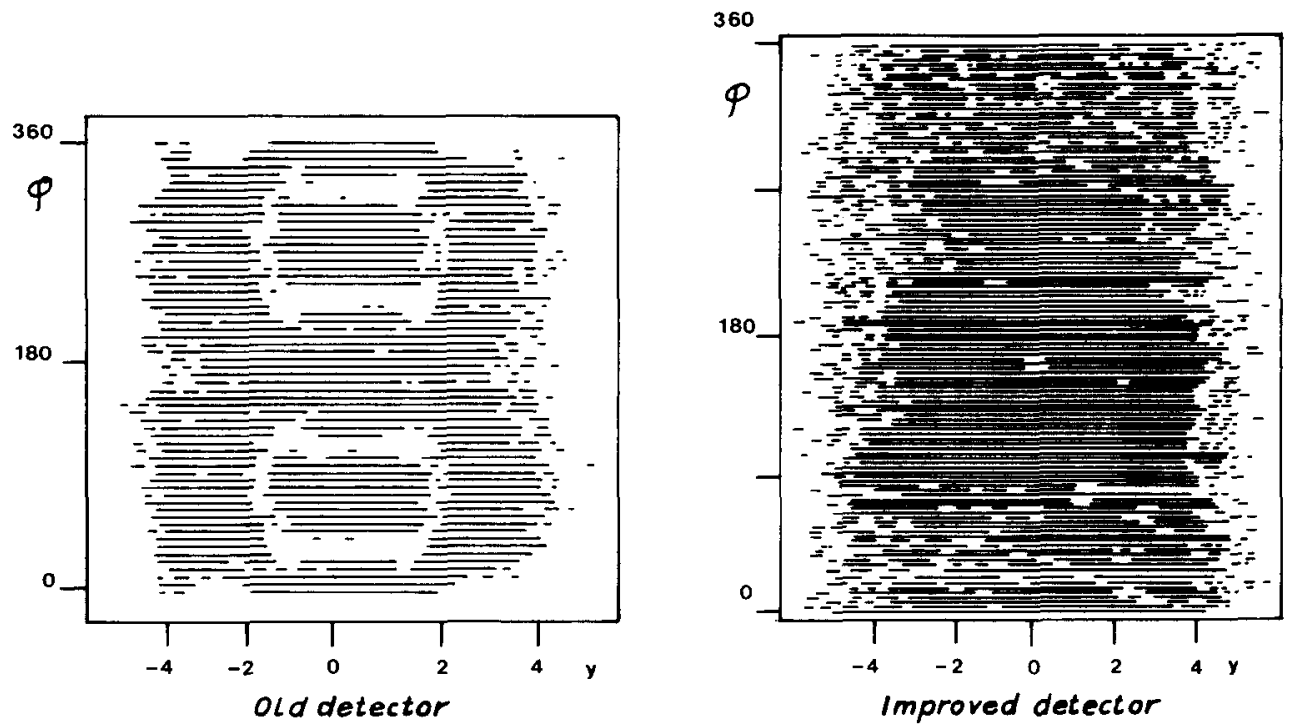

Acceptance as function of rapidity " $y$ "and azimutal production angle " $\varphi$ "

Fig. 4. Acceptance in the old (a) and the new (b) detector layout, as function of rapidity, $y$, and production angle, $\phi$. Fig. $4 \mathrm{~b}$ is based on simulated events ${ }^{6}$ ). b) A forward detection system with a total of 32 chamber units with two to eight wireplanes per unit.

A multigap proportional chamber, which provides position and energy loss measurement, has been developed and is included in the spectrometer $\left.{ }^{5}\right)$. The chamber, which has four gaps, covers a solid angle of about $2 \mathrm{sr}$. Its resolution on energy 


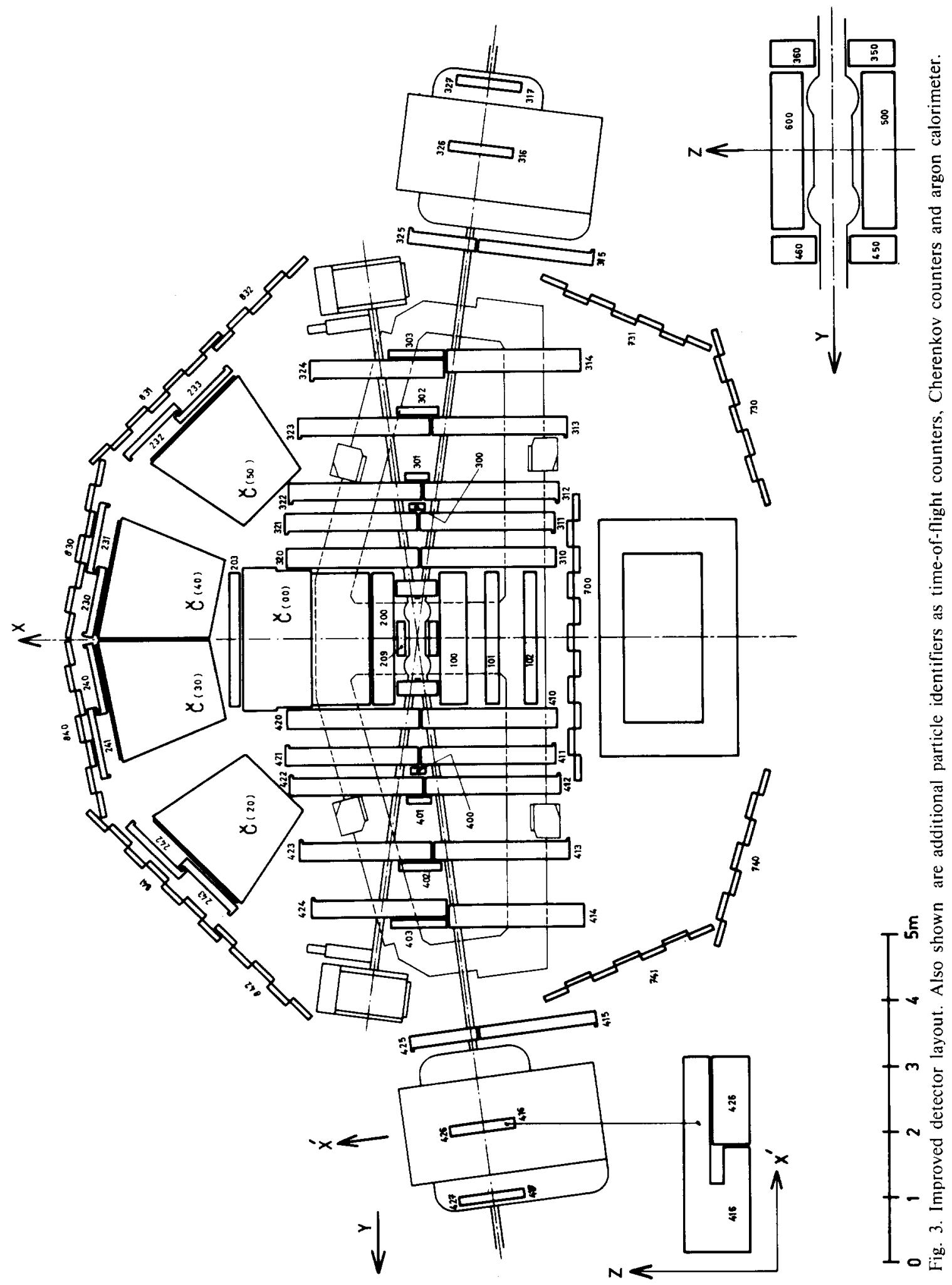


loss for minimum ionizing particles is $45 \% \mathrm{fwhm}$. The chamber can separate single electrons from close electron pairs. It can separate pions from protons up to $700 \mathrm{MeV} / c$.

\section{Chamber design}

The proportional chambers are of a foam-sandwich type. The mechanical design parameters and construction methods are given in ref. 1 . With the self-supporting sandwich plates the frame could be reduced to $5 \mathrm{~mm}$. This solution offers a large acceptance together with minimum weight. It also offers an easy way to stack together many plates to give a very compact detection unit. To increase the number of wireplanes in a limited space, it was sometimes needed to go from a standard gap width of $8 \mathrm{~mm}$ to $5 \mathrm{~mm}$. The additional density due to the detector inside the magnetic volume is about $0.024 \mathrm{~g} / \mathrm{cm}^{3}$.

A number of new wireplanes has been added. The effective area has been increased from $200 \mathrm{~m}^{2}$ to $300 \mathrm{~m}^{2}$ without any increase in the number of wires. This has been achieved by going from $2 \mathrm{~mm}$ wire spacing in the forward telescopes to $4 \mathrm{~mm}$. The corresponding change in the space resolution is from $\sim 0.7 \mathrm{~mm}$ to $\sim 0.9 \mathrm{~mm}^{4}$ ).

The acceptance losses, which were apparent in the old detector (fig. 4a), have been removed by additional chambers in the improved layout (fig. 4b).

We are indebted to E. Chesi and A. Minten for their contributions during the design phase.

We wish to thank our group of technicians, E. Albrecht, J. Cavalli, G. Cerutti, G. Gallay and J. Vinzia, for their continuous collaboration during the project.

\section{References}

1) R. Bouclier, G. Charpak, E. Chesi, L. Dumps, H. G. Fischer, H. J. Hilke, P. G. Innocenti, G. Maurin, A. Minten, L. Naumann, F. Piuz, S. C. Santiard and O. Ullaland, Nucl. Instr. and Meth. 115 (1974) 235.

2) R. Bouclier, R. C. A. Brown, E. Chesi, L. Dumps, H. G. Fischer, P. G. Innocenti, G. Maurin, A. Minten, L. Naumann, F. Piuz and O. Ullaland, Nucl. Instr. and Meth. 125 (1975) 235.

3) W. Bell, E. Chesi, R. Cooper, H. G. Fischer, G. Flügge, H. Frehse, Ch. Gottfried, B. Heck, P. G. Innocenti, I. McLaren, A. Minten, M. Scirè and D. Wegener, Nucl. Instr. and Meth. 124 (1975) 437.

4) H. G. Fischer, F. Piuz, W. J. Schwille, G. Sinapius and O. Ullaland, Proc. Int. Meeting on Proportional and drift chambers, Dubna (1975).

5) H. Frehse and F. Piuz, these proceedings.

6) D. Drijard, B. Heck and A. Norton, CERN-SFM note 3 (1977). 